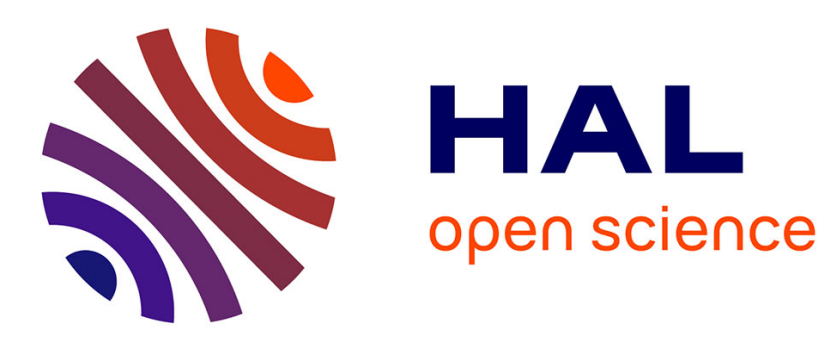

\title{
Effects of Energetic Heavy Ion Irradiation on the Structure and Magnetic Properties of FeRh Thin Films
}

\author{
N. Fujita, Y. Zushi, T. Matsui, A. Iwase, Y. Saito
}

\section{To cite this version:}

N. Fujita, Y. Zushi, T. Matsui, A. Iwase, Y. Saito. Effects of Energetic Heavy Ion Irradiation on the Structure and Magnetic Properties of FeRh Thin Films. The Seventh International Symposium on Swift Heavy Ions in Matter, Jun 2008, Lyon, France. hal-00257930

\section{HAL Id: hal-00257930 \\ https://hal.science/hal-00257930}

Submitted on 25 Jul 2008

HAL is a multi-disciplinary open access archive for the deposit and dissemination of scientific research documents, whether they are published or not. The documents may come from teaching and research institutions in France or abroad, or from public or private research centers.
L'archive ouverte pluridisciplinaire HAL, est destinée au dépôt et à la diffusion de documents scientifiques de niveau recherche, publiés ou non, émanant des établissements d'enseignement et de recherche français ou étrangers, des laboratoires publics ou privés. 


\title{
Effects of Energetic Heavy Ion Irradiation on the Structure and
}

\section{Magnetic Properties of FeRh Thin Films}

\author{
Nao. Fujita, Y. Zushi, T. Matsui, A. Iwase \\ Department of Materials Science, Osaka Prefecture University, Sakai, Osaka 599-8531,Japan \\ Y. Saito, \\ Japan Atomic Energy Agency(JAEA), Takasaki , Gumma, 370-1292, Japan \\ *corresponding author \\ phone $\quad 81-72-254-9810$ \\ e-mail iwase@mtr.osakafu-u.ac.jp
}

\begin{abstract}
Fe-54at.\%Rh thin films were irradiated with $10 \mathrm{MeV}$ iodine ions at room temperature.

Before and after the irradiations, the changes in magnetic properties and the lattice structure of the samples were studied by means of a SQUID magnetometer and X-ray diffraction. For the low fluence irradiation, the SQUID measurement at $20 \mathrm{~K}$ shows that the anti-ferromagnetic region of the thin film is changed into ferromagnetic region by the irradiation. As the film thickness is much smaller than the ion range, we can discuss
\end{abstract}


the relationship between the density of energy deposited by ions and the change in magnetization quantitatively. For the high fluence irradiation, the magnetization of the film is strongly decreased by the irradiation, which can be explained as due to the change in lattice structure from B2 into A1 structure by the irradiation.

Keywords: FeRh thin films, magnetic properties, energetic ion irradiation, lattice structure, SQUID

PACS code: $61.80 . \mathrm{Jh}, 61.82 . \mathrm{Bg}, 75.70 . \mathrm{Ak}$

\section{Introduction}

Equiatomic FeRh alloy, Fe-50at.\%Rh, with the B2 (CsCl type) structure shows a first order anti-ferromagnetic (AF)-ferromagnetic (FM) phase transition without any structural change [1-3]. In our previous studies, we have found that swift heavy ion irradiation induces ferromagnetic state in Fe-50at.\%Rh bulk alloy even at $20 \mathrm{~K}$ that is originally anti-ferromagnetic[4-7]. The ion range was, however, much smaller than the thickness of bulk FeRh samples. To study the effects of ion irradiation more clearly, we have to use FeRh thin films, thickness of which is much smaller than the range of irradiating ions. Under such an experimental condition, the change in ion energy in the sample is very small and the ions completely pass through the sample. Then, the ion 
energy can be deposited homogeneously in the specimen and we can discuss quantitatively the relationship between the deposited energy density (eV/cc) and the magnetization $(\mathrm{emu} / \mathrm{cc})$. Therefore, we have prepared FeRh thin films by means of an ion sputtering method, and have started the ion irradiation experiment using the thin films. This is our first report concerning the effects of energetic heavy ion irradiation on the structure and magnetic properties of FeRh thin films.

\section{Experimental procedure}

FeRh thin films about $200 \mathrm{~nm}$ thick were deposited on amorphous $\mathrm{SiO}_{2}$ substrates at room temperature by means of an ion beam sputtering from an alloy target of equiatomic FeRh. The base pressure of the ion sputtering chamber was about $6 \times 10^{-7} \mathrm{~Pa}$. After the deposition, the films were annealed at $600 \mathrm{C}$ for 4 hours under a pressure of 8 x $10^{-4} \mathrm{~Pa}$. The composition of the films was determined by an Electron Probe Microanalysis (EMPA).

The samples were irradiated with $10-\mathrm{MeV} \mathrm{I}$ ions to the fluence of $1 \times 10^{12}, 2 \times 10^{12}, 5$ $\times 10^{12}, 1 \times 10^{13}, 2 \times 10^{13}, 5 \times 10^{13}$ and $1 \times 10^{14} / \mathrm{cm}^{2}$ at room temperature using the tandem accelerator at JAEA-Takasaki. Before and after the irradiations, the temperature dependence of magnetization was measured with a SQUID magnetometer at $0.6 \mathrm{~T}$ in a 
temperature range of 5 to $320 \mathrm{~K}$. Structural changes were examined by using an X-ray diffraction method (XRD).

\section{Results and discussion}

The EPMA analysis has confirmed that the composition of the FeRh thin films was $\mathrm{Fe}: \mathrm{Rh}=46: 54$ on an average. Figure 1 shows the $\mathrm{XRD}$ spectrum for the annealed thin film sample. The spectrum apparently exhibits several diffraction peaks that correspond to the FeRh phase with $\mathrm{B} 2$ structure as well as to the A1 FeRh phase. (Here, A1 phase is known to be a fcc structure with a random distribution of iron and rhodium atoms). The relative peak intensity for the (nn0) planes of B2 phase is rather stronger than those for other lattice planes. The XRD result has revealed that the annealed FeRh thin film samples are composed of two phases: the (110) preferentially oriented B2-type FeRh and the Al-type FeRh phase. Figure 2 shows the magnetization versus temperature (M-T) curves of the annealed FeRh thin films and their XRD profiles were shown in fig.1. It should be noted, here, that the A1-type FeRh phase does not contribute to the total magnetic moment in the present M-T measurement. These facts will be discussed later in this paper. As clearly seen in the figure, the film exhibits the AF-FM phase transition behavior in the temperature between $100-300 \mathrm{~K}$. Several papers have reported 
so far that thin films of FeRh exhibit a broad and incomplete magnetic phase transition, and a large thermal hysteresis in contrast to bulk FeRh samples[8-12]. The similar tendencies are observed in our present samples. The value of the magnetization of the film below the phase transition temperatures is still finite implying that only some part of the B2-phase exhibits AF-FM transition, and the rest of the B2-phase is still ferromagnetic at low temperature. The films prepared in the present studies, however, show a clear AF-FM magnetic transition. Therefore, ion-irradiation effects on the magnetic transition of the FeRh films can be definitely evaluated by using the present thin film samples. Hence, we performed the ion irradiation experiments using these thin film samples.

We discuss first of all, the experimental results for the low ion-fluence irradiation (below $5 \times 10^{12} / \mathrm{cm}^{2}$ ). M-T curves of the FeRh thin films before and after irradiations for the low ion-fluence are shown in Fig.3(a). For clarity, in the figure, we show only the M-T curves measured during heating up the sample from $5 \mathrm{~K}$ because both $\mathrm{M}-\mathrm{T}$ curves during heating and cooling show the same trend about the effect of irradiation. Fig.3(b) shows the values of saturated magnetization (Ms) at $20 \mathrm{~K}$ and $320 \mathrm{~K}$ for the low fluence irradiations as a function of deposited energy density. As can be seen in the figure, the magnetization below the magnetic transition temperature increases and the transition 
temperature shifts to a lower temperature side with increasing the ion-fluence. The magnetization of the film exhibits the maximum value for the ion-fluence of $5 \times 10^{12} / \mathrm{cm}^{2}$, where the FM-AF phase transition completely disappears. The tendency of the increase in Ms of FeRh thin films by the ion irradiation is the same as that of bulk FeRh samples irradiated with $100-200 \mathrm{MeV}$ heavy ions[4-7]. The fluence where the magnetization shows the maximum value for the thin films is different from that for bulk FeRh, because it should strongly depends on the kinds and the energy of ions. On the other hand, above the transition temperature, the magnetization of FeRh thin films does not change even when irradiated up to the fluence of $5 \times 10^{12} / \mathrm{cm}^{2}$. Fig. 4 shows the ion-fluence variation of XRD spectra for FeRh thin films. The XRD spectra for the low fluence (below $5 \times 10^{12} / \mathrm{cm}^{2}$ ) are about the same as for the unirradiated sample, meaning that the lattice structure is scarcely changed by the irradiation. Figures 3 and 4 indicate that above the transition temperature, the magnetic property and the lattice structure of the ferromagnetic B2 phase region is little affected by the irradiation, while below the transition temperature, the anti-ferromagnetic phase region with a B2 structure is changed into ferromagnetic by the irradiation. At the fluence of $5 \times 10^{12} / \mathrm{cm}^{2}$, where Ms shows the maximum value, all B2 structured region becomes ferromagnetic. As the irradiation induced ferromagnetism lasts for one month or more after the irradiation, 
atomic arrangement in FeRh B2 structure is partly modified by the irradiation nearly permanently. But this modification is too small to change the B2 structure. The reason why this small modification of atomic arrangement can induce the ferromagnetism still remains uncertain

Next, the result for the high ion-fluence irradiation $\left(1 \times 10^{13}-1 \times 10^{14} / \mathrm{cm}^{2}\right)$ is discussed. Fig. 5a shows M-T curves of FeRh thin films for the high fluence irradiations and the values of Ms at $20 \mathrm{~K}$ and $320 \mathrm{~K}$ are plotted as a function of deposited energy density in Fig. 5b. As can be seen in Fig. 5, the magnetization of the film is strongly decreased by the irradiation. The height of diffraction peaks corresponding to the B2 structure in the $\mathrm{XRD}$ spectrum for the fluence of $2 \times 10^{13} \mathrm{ion} / \mathrm{cm}^{2}$ is smaller than that for unirradiated sample. When the sample is irradiated up to the fluence of $1 \times 10^{14} / \mathrm{cm}^{2}$, we can not see any diffraction peaks for the B2 structure, and only peaks for A1 structure are observed (see Fig.4). This fact indicates that the B2 structure is changed into the A1 structure by irradiation with high ion-fluence.

From the experimental result described above, we can summarize the changes in the lattice structure and magnetic state of FeRh thin films by the $10 \mathrm{MeV}$ I ion irradiation as follows; unirradiated $\mathrm{FeRh}$ thin films have three-phases (ferromagnetic B2 structured region, anti-ferromagnetic B2 structured region and nonmagnetic A1 structured region) 
below the FM-AF magnetic transition temperature. For low ion-fluence, the irradiation gradually changes anti-ferromagnetic B2 structured region into ferromagnetic B2 structured region. All B2 structured region becomes ferromagnetic at the ion-fluence of $5 \times 10^{12} / \mathrm{cm}^{2}$. For higher ion-fluence, ferromagnetic B2 structure is changed into nonmagnetic A1 structure, causing a strong decrease in Ms.

Through the present experiment, we could clearly observe the $10 \mathrm{MeV}$ I irradiation effects of FeRh thin films. As a next step, we plan to discuss quantitatively the dependence of magnetic property change on some irradiation parameters ( ion energy, electronic and nuclear stopping powers, ion velocity and so on) by conducting a variety of irradiation experiments.

\section{Summary}

Fe-54at.\%Rh thin films were irradiated with $10 \mathrm{MeV}$ I ions at room temperature. Magnetization versus temperature curves, the changes in the saturated magnetization and structural changes have been studied after the irradiation. For low ion-fluence irradiation, the magnetization increases and the magnetic transition point shifts a lower temperature side without any change in the lattice structure. For high ion-fluence, magnetization decreases because B2 structure becomes A1 structure. 
By using the FeRh thin films, the relationship between the deposited energy density and change in magnetization could be quantitatively discussed.

\section{Acknowledgments}

This research has partially been supported by the Reimei Research Promotion project (Japan Atomic Energy Agency)

\section{References}

[1]M. Fallot, R. Hocart, Rev. Sci., 498 (1939)

[2]J. S. Kouvel, and C. C. Hartelius, J. Appl. Phys. Suppl. 33, 1343-1344 (1962)

[3]J. S. Kouvel, J. Appl. Phys. 37, 1257 (1966)

[4]M. Fukuzumi, Y. Chimi, N. Ishikawa, F. Ono, S. Komatsu, and A. Iwase, Nucl. Instr. and Meth. B230,269-273 (2005)

[5]M. Fukuzumi, Y. Chimi, N. Ishikawa, M. Suzuki, M. Takagaki, J. Mizuki, F. Ono, R.Neumann, and A. Iwase, Nucl. Instr. and Meth. B245,161-165 (2006)

[6]A. Iwase, M. Fukuzumi, Y. zushi, M. Suzuki, M. Takagaki, N. Kawamura, Y. Chimi, N. Ishikawa, J. Mizuki, and F. Ono, Nucl. Instr. and Meth. B 256, 429 (2007) 
[7]Y. Zushi, M. Fukuzumi, Y. Chimi, N. Ishikawa, F. Ono, and A. Iwase, Nucl. Instr. and Meth. B 256,434 (2007)

[8]J. M. Lommel, J. Appl. Phys. 37, 1483-1484 (1966)

[9]J. M. Lommel and J. S. Kouvel, J. Appl. Phys. 38, 1263 (1967)

[10] Y. Ohtani and I. Hatakeyama, J. Appl. Phys. 74, 3328-3332 (1993)

[11] Y. Ohtani and I. Hatakeyama, J. Magn. Magn. Mater. 131, 339-344 (1994)

[12] S. Hashi, S. Yanase, Y. Okazaki, and M. Inoue, IEEE. 40 ,5, 2784-2786 (2004) 


\section{Figure captions}

Fig. 1 XRD profile for unirradiated Fe-54at.\%Rh thin film.

Fig. 2 Magnetization versus temperature for unirradiated Fe-54at.\%Rh thin film.

Fig. 3 (a) M-T curves of the FeRh thin films before and after irradiations for the low ion-fluence $\left(<5 \times 10^{12} \mathrm{ion} / \mathrm{cm}^{2}\right)$ (b) Values of saturated magnetization (Ms) as a function of deposited energy density at $20 \mathrm{~K}$ and $320 \mathrm{~K}$.

Fig.4 Normalized XRD profiles of Fe-54at.\%Rh thin films (a) uniradiated and irradiated with ion fluence of (b) $1 \times 10^{12} \mathrm{ion} / \mathrm{cm}^{2}$, (c) $5 \times 10^{12} \mathrm{ion} / \mathrm{cm}^{2}$,(d) $2 \times 10^{13} \mathrm{ion} / \mathrm{cm}^{2}$ and (e) $1 \times 10^{14}$ ion $/ \mathrm{cm}^{2}$.

Fig. 5 (a) M-T curves of the FeRh thin films before and after irradiations for the high ion-fluence $\left(>5 \times 10^{12} \mathrm{ion} / \mathrm{cm}^{2}\right)$ (b) Values of saturated magnetization (Ms) as function of deposited energy density $\left(>\times 10^{19} \mathrm{keV} / \mathrm{cc}\right)$ at $20 \mathrm{~K}$ and $320 \mathrm{~K}$. 


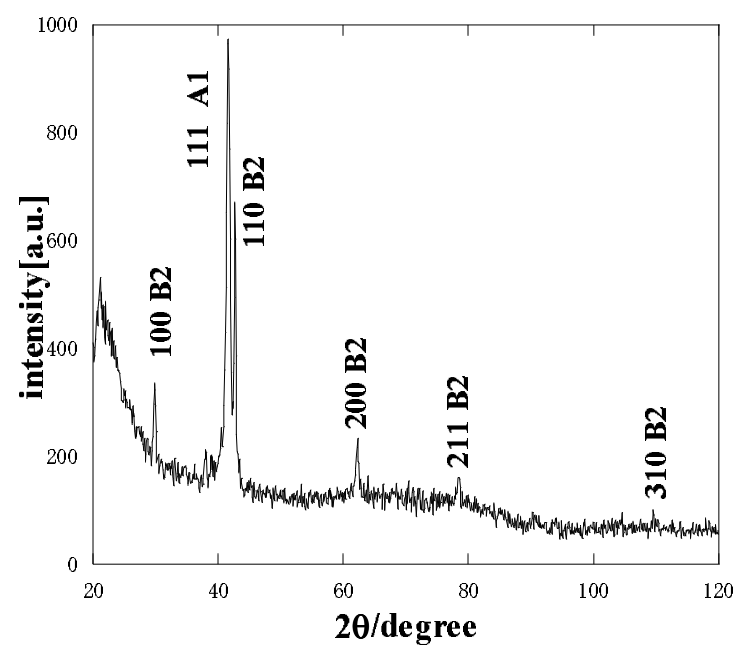

Fig.1 (Nao. Fujita) 


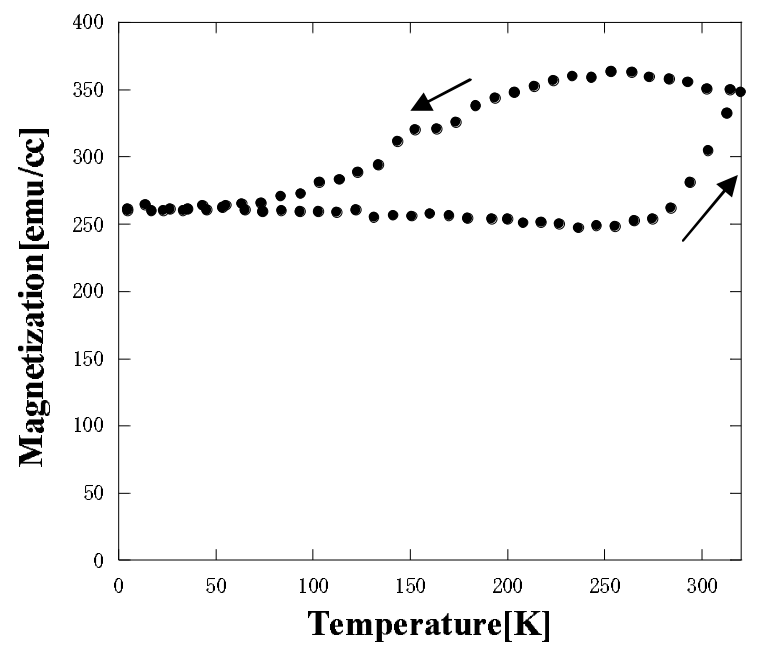

Fig. 2 (Nao. Fujita) 

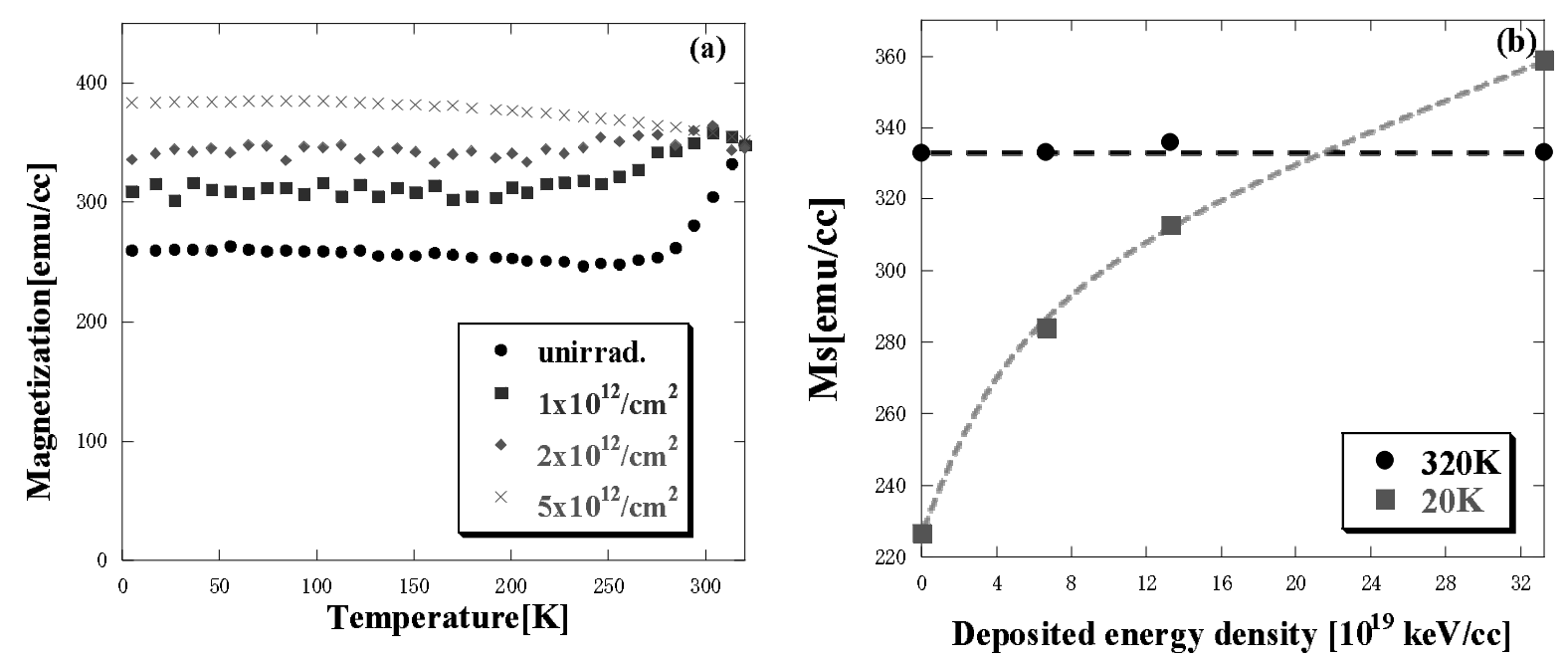

Fig. 3 (Nao. Fujita) 


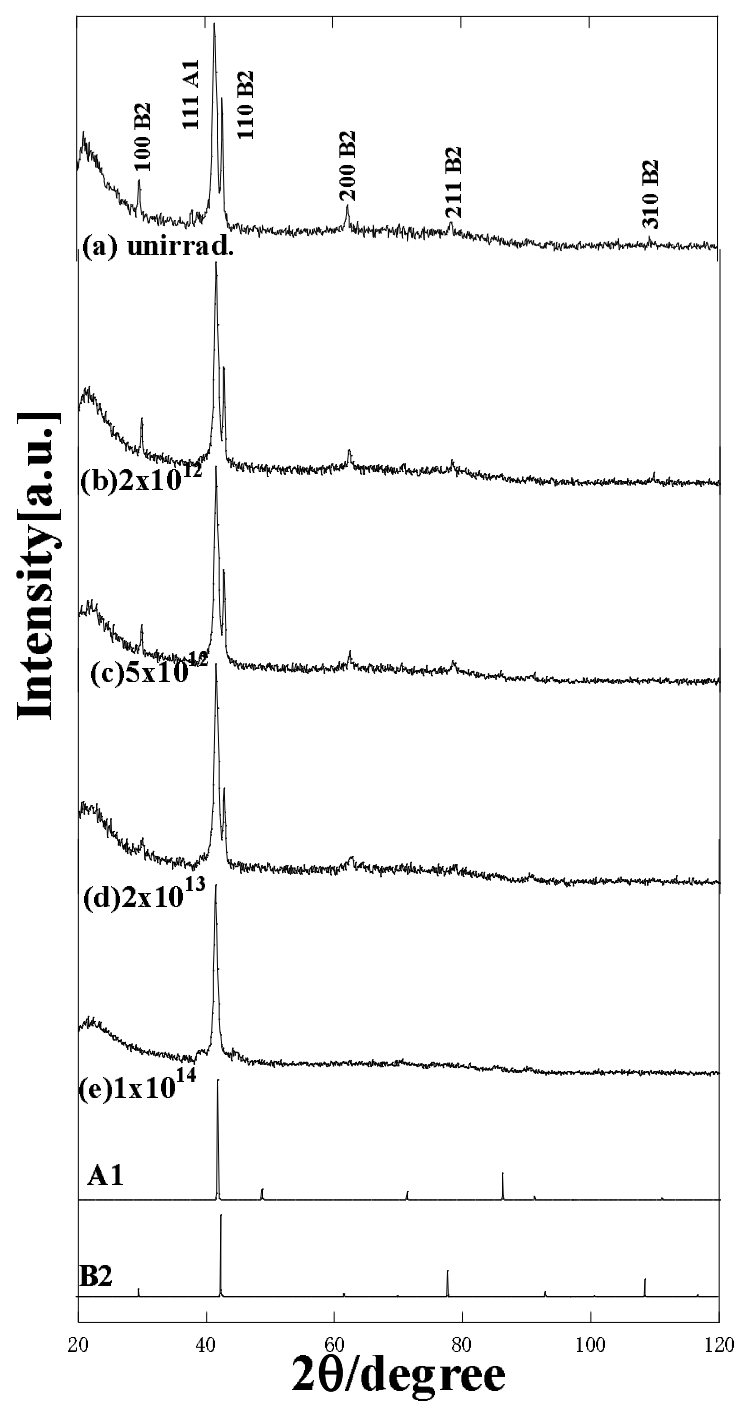

Fig.4 (Nao. Fujita) 

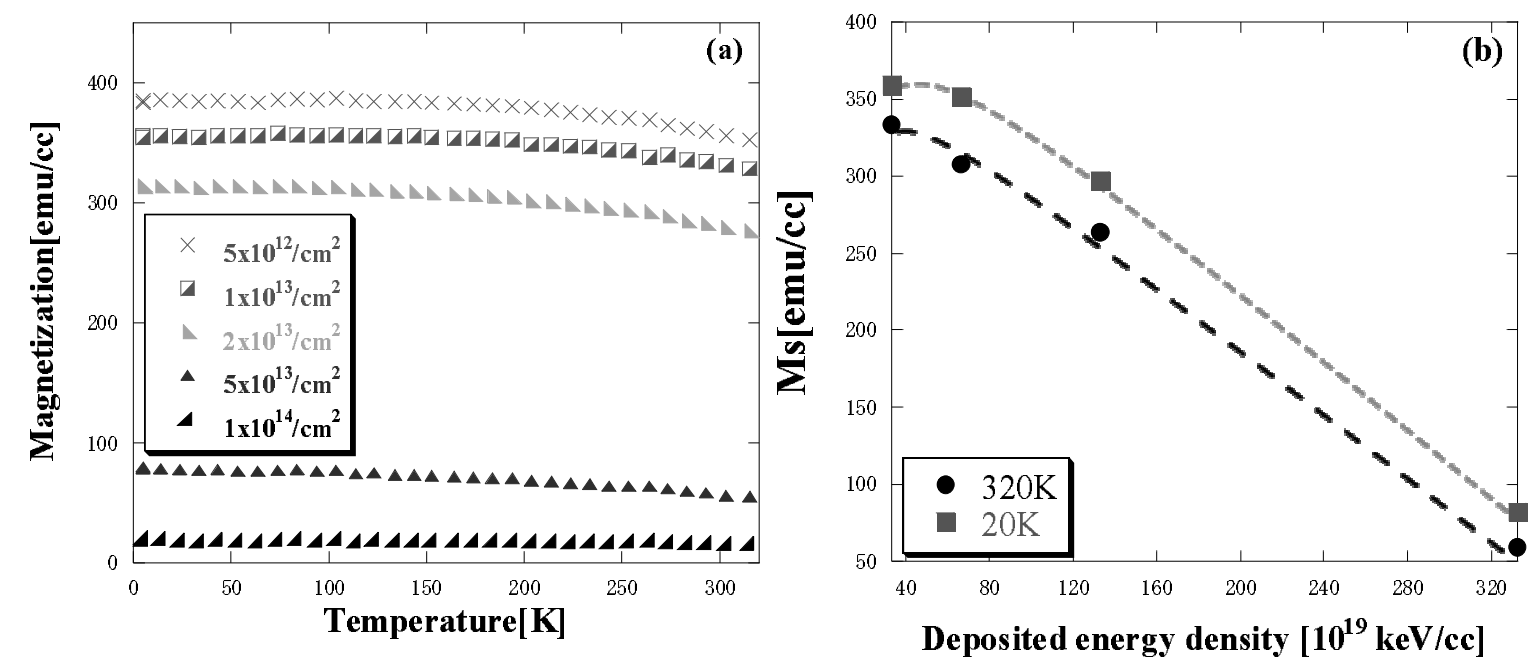

Fig.5 (Nao. Fujita) 